\title{
The Real Cost of Theoretic Risk Avoidance: The Need to Challenge Unsubstantiated Concerns About ${ }^{131}$ I Therapy
}

$\mathbf{F}$

or many years, the Nuclear Regulatory Commission in the United States required hospitalization for purposes of radiation isolation of patients receiving $1.11 \mathrm{GBq}(30 \mathrm{mCi})$ or more of ${ }^{131}$ I. Furthermore, admission for purposes of radiation isolation was required even if the patient was an adult who lived alone or had a separate bedroom and bathroom and was capable of self-care. Once admitted, these patients remained hospitalized until radiation safety personnel confirmed that the radiation emitted from the patient was less than $5 \mathrm{mR} / \mathrm{h}$ at $1 \mathrm{~m}$. This criterion often resulted in an overnight stay, but patients who received doses

\section{See page 683}

of $3.7 \mathrm{GBq}(100 \mathrm{mCi})$ or more and older patients whose renal excretion of the ${ }^{131}$ I was impaired often required 2 or more overnight stays. Although this practice offered a respite from job and household chores for some patients, others complained about the inconvenience associated with this hospitalization and the consequent loss of many personal conveniences. In addition, the admission required occupancy of a single room with toilet facilities, preferably in a building of older, more substantial construction, possibly a corner room to avoid potential exposure of patients in an adjoining room or preferably a room adjacent to an unoccupied resource

Received Feb. 22, 2011; revision accepted Feb. 25, 2011

For correspondence contact: Stanley J. Goldsmith, New York Weill Cornell Medical Center, 525 E. 68th St., Starr-221, New York, NY 10021.

E-mail: sjg2002@med.cornell.edu

COPYRIGHT @ 2011 by the Society of Nuclear Medicine, Inc.

DOI: 10.2967/jnumed.110.081927 such as an electrical or supply closet or a stairwell.

In Europe and other jurisdictions, the requirement for isolation was established for even lower doses, even as low as $185 \mathrm{MBq}(5 \mathrm{mCi})$ of ${ }^{131} \mathrm{I}$. In certain cases, the facilities had isolated plumbing and storage tanks for bodily wastes. All these details involve incremental costs and compete with other necessary hospital and societal services.

In 1997, after considerable examination of commentary presented by knowledgeable sources, the Nuclear Regulatory Commission revised title 10 of Code of Federal Regulations part 35.75 to permit release of patients treated with ${ }^{131} \mathrm{I}$ in doses greater than 1.11 $\mathrm{GBq}$ provided that no other individual was likely to be exposed to more than 5 $\mathrm{mSv}$. This seemed at the time a reasonable response to the concerns about the incremental costs associated with hospitalization and the evidence that, in practice, the exposure of any individual was not likely to exceed the specified level, a level considered to be far below an absorbed dose that is apt to have consequences for the exposed individual. Despite the logic of this reasoning, there was, and continues to be, opposition by a variety of individuals to releasing patients who have received ${ }^{131}$ I. Furthermore, many national regulatory agencies throughout Europe, Asia, and elsewhere continue to require hospitalization and isolation of patients treated with ${ }^{131} \mathrm{I}$ and other radioactive materials.

According to the linear nonthreshold hypothesis, exposure to radiation at any level is apt to produce radiationrelated disease, most commonly interpreted to be the development of malignancy-even though many epidemiologic studies fail to conclusively demonstrate low-dose radiation as a causal factor in development of malig- nancy. Many radiation epidemiologists support the notion of the linear nonthreshold hypothesis, but the validity of this hypothesis cannot be proven. As a worst-case hypothesis, it is useful to determine reasonably safe exposure limits, assess the costs involved in further reducing the exposure of an individual or groups of individuals, and compare the negligible benefits obtained with the incremental costs involved. Are the funds expended to reduce or eliminate exposure of members of a patient's family or the public a worthwhile use of resources to further reduce a theoretic risk that already approaches zero? Or are the alwayslimited resources better spent in providing more nursing care for those in need, food for the hungry, education for children, better and less costly transportation for workers, and other societal services?

In Japan, the regulations governing the use of radioactive materials continue to require hospital admission and isolation of patients receiving ${ }^{131} \mathrm{I}$ therapy. The costs of doing so are significant, and there are a limited number of hospital beds appropriate for this purpose. Inevitably, as reported by Higashi et al. in this issue of the Journal of Nuclear Medicine (1), the restrictions have led to a backlog of patients requiring this therapy, resulting in delays in hospitalization and treatment that in some instances have exceeded $180 \mathrm{~d}$ from the time that the indication for ${ }^{131}$ I was determined. In their retrospective study of 198 patients with metastatic thyroid carcinoma (47 patients with lymph node metastases only, 105 with lung metastases, and 46 with bone and other distant metastases), Higashi et al. found that 24 patients died from thyroid carcinoma within an average follow-up period of $5.37 \mathrm{y}$. Among the variables 
in a multivariate analysis, a delay in ${ }^{131}$ I treatment of $180 \mathrm{~d}$ or more was associated with a 4.22 times increase in the risk of death. This is a startling and chilling observation that confirms the real harm associated with depriving or delaying ${ }^{131} \mathrm{I}$ therapy. The delays in Japan are an inevitable consequence of medical economics, which exist throughout the world. We are no longer talking about a theoretic incremental risk on the order of a small fraction of the natural incidence of malignancy but a greater than 4fold increase in death from disease as a consequence of treatment delays that evolved from overregulation of ${ }^{131} \mathrm{I}$ therapy and restricted access to expensive and otherwise unnecessary resources mandated not on the basis of available scientific facts but simply because of unfounded anxiety and political decision making.

After the 1997 regulatory modification allowing release of patients receiving more than $1,110 \mathrm{MBq}$ of ${ }^{131} \mathrm{I}$ - provided the nuclear practitioner has demonstrated to the licensing authority that patients have been instructed on reasonable isolation and that conditions are such that no member of the public is likely to be exposed beyond $500 \mathrm{mSv}$-Grigsby et al. (2) distributed radiation-monitoring devices to family members (adults, children, and pets) of patients receiving 3.7-5.5 GBq of ${ }^{131} \mathrm{I}$. None of those evaluated received more than $20 \%$ of the allowable absorbed dose. More recently, Pant et al. (3) assessed the radiation dose to family members of patients receiving ${ }^{131} \mathrm{I}$ therapy for hyperthyroidism or thyroid cancer. Although the doses administered to hyperthyroid patients are considerably lower than the doses used to ablate or treat patients with thyroid carcinoma, hyperthyroid patients retain a considerably greater fraction of the administered dose and thus represent a potential radiation source for a longer period. Among 45 hyperthyroid patients receiving $185-500 \mathrm{MBq}$ of ${ }^{131} \mathrm{I}$, family members received a measured absorbed dose of $0.4-2.4 \mathrm{mSv}$ (mean, $1.1 \mathrm{mSv}$ ). Twenty-three of the 45 patients were advised to sleep in separate beds for 3 nights. The absorbed dose in this group ranged from 0 to 1.9 $\mathrm{mSv}$ (mean, $0.6 \mathrm{mSv}$ ).

Among 297 family members of thyroid carcinoma patients treated with $0.925-7.4 \mathrm{GBq}$ and released to a variety of living conditions, the mean exposures were $0.4-0.8 \mathrm{mSv}$, with only a few individuals receiving greater than $5.0 \mathrm{mSv}$.

In the United States, some individuals and political representatives continue to seek reversal or revision of the current regulations that allow patient release after ${ }^{131} \mathrm{I}$ therapy. One of the stated concerns is the potential exposure of hotel workers in the event a patient elects to occupy a hotel room for several days after ${ }^{131}$ I therapy, either by choice or out of concern for family members because of specific housing limitations. The various hearsay, undocumented reports of excessive exposure of individuals occupying hotel rooms subsequent to occupancy by ${ }^{131}$ I-treated individuals has led to a reexamination by the Nuclear Regulatory Commission's Advisory Committee on Medical Use of Isotopes of the potential exposure to individuals in a variety of circumstances who might come into contact with ${ }^{131}$ I-treated patients (4). In no instance in any realistic and even hyperbolic scenario is an individual likely to receive radiation exposure in excess of a level deemed exceedingly safe.

In a recent commentary, Kloos (5) presented a rational examination of the evidence related to consequences from exposure to patients who have received ${ }^{131} \mathrm{I}$. Except for pregnant or nursing patients, in whom there are clear consequences to the unborn or nursing infant, Kloos states that "the harm that a radioiodine-treated patient could inflict on another person while following common sense instructions appears to be low" and certainly is undocumented.

As nuclear medicine practitioners, we want to continue to be able to provide the benefits of radioactivity in general to patients and to do so in a manner that is safe for patients, their families, our staff, and ourselves. There is ample evidence that ${ }^{131}$ I-treated patients can be released with prudent advice about potential exposure to others and without injury to family members, hotel workers, or the general public. It is important that we continue to challenge unsubstantiated concerns that might interfere with patient access to ${ }^{131} \mathrm{I}$ and the adverse consequences that result from treatment delays as demonstrated by the report from Higashi et al. (1).

\section{Stanley J. Goldsmith}

New York-Presbyterian Hospital and

Weill Medical College of Cornell

University

New York, New York

\section{REFERENCES}

1. Higashi T, Nishii R, Yamada S, et al. Delayed initial radioactive iodine therapy resulted in a poor survival in patients with metastatic differentiated thyroid carcinoma: a retrospective statistical analysis of 198 cases. J Nucl Med. 2011;52:683-689.

2. Grigsby PW, Siegel BA, Baker S, Eichling JP. Radiation exposure from outpatient radioactive iodine $\left({ }^{131} \mathrm{I}\right)$ therapy for thyroid carcinoma. JAMA. 2000;283:2272-2274.

3. Pant GS, Sharma SK, Bal CS, Kumar R, Rath GK. Radiation dose to family members of hyperthyroidism and thyroid cancer patients treated with ${ }^{131} \mathrm{I}$. Radiat Prot Dosimetry. 2006;118:22-27.

4. Fisher D, Gilley D, Langhorst S, et al. Advisory Committee on the Medical Use of Isotopes (ACMUI): Patient Release Report-December 13, 2010 Available at: http://adamswebsearch2.nrc. gov/idmws/DocContent.dll?library $=$ PU_ADAMS ${ }^{\wedge}$ pbntad01\&LogonID =a04e47b69c822268ae 178 6d773f963c4\&id=103490039. Accessed March 7, 2011

5. Kloos RT. Survey of radioiodine therapy safety practices highlights the need for user-friendly recommendations. Thyroid. 2011;21:97-99. 